\title{
The Complex Structure of 2D Surfaces
}

\author{
H.Kawai, N.Tsuda and T.Yukawa \\ National Laboratory for High Energy Physics (KEK), Tsukuba 305, Japan
}

\begin{abstract}
The complex structure of a surface generated by the two-dimensional dynamical triangulation(DT) is determined by measuring the resistivity of the surface. It is found that surfaces coupled to matter fields have well-defined complex structures for cases when the matter central charges are less than or equal to one, while they become unstable beyond $c=1$. A natural conjecture that fine planar random network of resistors behave as a continuous sheet of constant resistivity is justified numerically for $c<1$.
\end{abstract}

\section{Introduction}

The discretized approach of 2D quantum gravity, implemented by the matrix model, exhibits, in a continuum limit the same behavior as the continuous approach, given by the Liouville field theory. Thus it is certain that the two theories describe the same physics. However, we feel that the mutual relationship is not yet fully understood. While the relation between DT and matrix model is transparent, its relation to the Liouville field theory is not so evident. Intuitively, we expect that the simplicial manifolds generated by DT will tend to continuous manifolds in the large number limit of simplexes. However, such surfaces are known to be fractal[1], hence the way the lattice regularization simulates the continuous theory is not at all clear. It is therefore indispensable to make sure that an universal continuum limit exists. In order to check this numerically, we propose to measure how the network conducts currents, and compare it with the corresponding continuous medium. An invariance under local scale transformation plays an essential role in the algorithm, and because of this property we can extract information about the complex structure which is independent of the conformal mode parametrised by moduli.

Despite many theoretical developments the precise nature of the transition at $c=1$ is still not fully understood. Serious numerical efforts have failed so far to convincingly establish the transition, at least by measuring the string susceptibility. It should be much easier to observe this transition in local scale invariant quantities, rather than in the original metric which is comparatively complicated reflecting the fractal nature of the surface. Here, we show such a method by which we can define the complex structure of a DT surface and separate the conformal mode.

\section{Determination of the complex structure}

The basic idea of our method originates from the analog model [2] of the dual amplitude in analogy with the electrical circuit [3]. Let us first consider a two dimensional conducting medium with conductivity tensor density $\sigma^{\mu \nu}$. Ohm's law reads

$j^{\mu}=\sigma^{\mu \nu} E_{\nu}$,

where $E_{\nu}\left(=-\partial_{\nu} V\right)$ is an electric field and $j^{\mu}$ is a current density. The Joule heat $Q$ can be estimated by a potential distribution $V$ on the surface; $Q=\int d^{2} x \sigma^{\mu \nu} \partial_{\mu} V \partial_{\nu} V$. Requiring $\delta Q=0$, it leads to an equation for $V$ : $\partial_{\mu} \sigma^{\mu \nu} \partial_{\nu} V=0$, which is just the equation of continuity. For a homogeneous isotropic medium the conductivity tensor density is given by $\sigma^{\mu \nu}=\frac{1}{r} \sqrt{g} g^{\mu \nu}$ with $r$ being the resistivity constant. Therefore, Ohm's law(11) is invariant under the local scale transformation $g_{\mu \nu} \mapsto g_{\mu \nu} e^{-\sigma}$. In the following we first give an algorithm to measure the resistivity from voltage distribution. In the case of spherical topology we can regard the surface essentially as an infinite flat sheet. Then the potential at a point specified by the complex coordinate, $z$, with a source of current $I$ placed at $z_{k}$, and a sink of 
the current at $z_{l}$ is given by

$V(z)=-\frac{I r}{2 \pi} \ln \left|\frac{z-z_{k}}{z-z_{l}}\right|+$ Const.

In order to avoid the ambiguity arising from Const. in eq.(2) we measure the potential drop between coordinates $z_{i}$ and $z_{j}$. We define the potential drop between points $i$ and $j$ in the presence of a source and a sink of current at points $k$ and $l$ by

$$
\begin{aligned}
V_{i j}^{(k)(l)} & \equiv V\left(z_{i}\right)-V\left(z_{j}\right) \\
& =-\frac{I r}{2 \pi} \ln \left|\left[z_{i}, z_{j} ; z_{k}, z_{l}\right]\right|
\end{aligned}
$$

where $\left[z_{i}, z_{j} ; z_{k}, z_{l}\right]=\frac{z_{i}-z_{k}}{z_{i}-z_{l}} \frac{z_{j}-z_{l}}{z_{j}-z_{k}}$ is known as the anharmonic ratio. It has an important property: i.e. $S L(2, C)$ invariance. Then it allows us to fix three coordinates among the $\left\{z_{i}, z_{j}, z_{k}, z_{l}\right\}$ to any desired values without changing the potential drop eq.(3). For example, we can fix three coordinates as $z_{j}=1, z_{k}=0$, and $z_{l}=\infty$. Then the potential drop eq.(3) for $I=1$ is written as

$$
\begin{aligned}
V_{i j}^{(k)(l)} & =-\frac{r}{2 \pi} \ln \left|z_{i}\right|, \\
V_{i k}^{(j)(l)} & =-\frac{r}{2 \pi} \ln \left|1-z_{i}\right| .
\end{aligned}
$$

All the other possible permutations of $\{z\}$ only give the potential drops obtained by linear combinations of eq.(4) and eq.(5). Since unknown variables are $\left\{z_{i}, r\right\}$, we need the potential drop at an additional point $z_{m}$. Then we get three more relations in addition to eq.(4) and eq.(5) which altogether are sufficient to specify five unknowns: $\left\{z_{i}, z_{m}, r\right\}$. The three extra equations are given by

$V_{m j}^{(k)(l)}=-\frac{r}{2 \pi} \ln \left|z_{m}\right|$

$V_{m k}^{(j)(l)}=-\frac{r}{2 \pi} \ln \left|1-z_{m}\right|$,

$V_{i k}^{(m)(l)}+V_{m j}^{(k)(l)}=-\frac{r}{2 \pi} \ln \left|z_{i}-z_{m}\right|$.

Now we apply this to a random surface generated by DT. Since the local scale invariance is not fully realized on this surface, the resistivity may have certain fluctuations. In fact, the value varies from one sample to another, and depending on the position of electrodes for the measurements. Such ambiguities should, however, vanish in the continuum limit, if it exists at all. The dual graph of a surface consisting of $N$ triangles is regarded as a trivalent network, where we fix the resistance of the link connecting two neighbouring vertices to be 1 . What we want to do is to examine whether such a network behaves like a continuous medium in the limit of $N \rightarrow \infty$. For the measurement we pick five vertices in the dual graph and perform the method as explained above. The practical method we employ for the determination of potential drops is as follows; we pick two vertices at $v_{\text {in }}$ and $v_{\text {out }}$ for the source and the sink of current with unit intensity. By writing the potential of the vertex $v$ as $V(v)$, current conservation reads $V(v)=\frac{1}{3}\left\{\sum_{i=1,2,3} V\left(v_{i}\right)+\delta_{v, v_{\text {in }}}-\delta_{v, v_{\text {out }}}\right\}$, where $v_{1}, v_{2}$ and $v_{3}$ are the three neighboring vertices of $v$. We solved this set of equations by the successive overrelaxation method.

\section{Analog model}

Our method can be reconsidered from the string-theoretical point of view. Whether string theory can be described by local fields or not is one of the fundamental questions. The authors of 22 showed that a sum of planar fishnet Feynman diagrams with $N$ external particles is indeed approximated by a $N$ particle Veneziano amplitude. The basic assumption of this model is that the network corresponding to a fine planar fishnet Feynman diagram can be regarded as a homogeneous isotropic conducting sheet with a constant resistivity. Once this is accepted it is straightforward to derive dual amplitudes by evaluating the heat generated by the sheet. The purpose of our article is to justify this basic assumption numerically. In order to clarify the correspondence to the network of resistors, we write the amplitude of fishnet Feynman diagrams as

$$
\sum_{\substack{\text { planar } \\ \text { diagrams }}} \int \prod_{l: \text { propagator }} d \alpha_{l} \rho\left(\alpha_{l}\right) \int \prod_{i: \text { vertex }} d^{D} x_{i} e^{-Q}(9)
$$


where $Q=\sum_{l} \frac{\left(x_{i}-x_{j}\right)^{2}}{2 \alpha_{l}}, l$ and $(i, j)$ indicate a link and its two ends(vertices) of the network respectively, and $\rho\left(\alpha_{l}\right)=\frac{1}{2(2 \pi)^{D / 2}} \alpha_{l}^{-\frac{D}{2}} e^{-\frac{1}{2} \alpha_{l} m^{2}}$. Here we have introduced a Feynman parameter $\alpha_{l}$ f for each propagator $l$ in the momentum integration,

$$
\begin{aligned}
& \int \frac{d^{D} p}{(2 \pi)^{D}} \frac{1}{p^{2}+m^{2}} e^{i p(x-y)} \\
& \quad=\int_{0}^{\infty} d \alpha_{l} \frac{1}{2(2 \pi)^{D}} \alpha_{l}^{-\frac{D}{2}} e^{-\frac{1}{2} \alpha_{l} m^{2}} e^{-\frac{(x-y)^{2}}{2 \alpha_{l}}}
\end{aligned}
$$

The basic conjecture is that the fishnet diagrams behaves as a continuum homogeneous conducting sheet after taking the average $\sum_{\text {diagrams }} \int \prod_{l: \text { propagator }} d \alpha_{l} \rho\left(\alpha_{l}\right)$.

\section{Numerical results and discussions}

We fix the topology to be two sphere $\left(S^{2}\right)$ and the number of triangles to be 4,000, 8,000 and 16,000 (prohibiting tadpole and self-energy diagrams). For matter fields coupled to gravity we put $n$ scalar fields with $n=0,1,2$ and 3 and/or Ising spins on each triangle.

\subsection{Resistivity distributions}

We pick-up five vertices randomly as five electrodes to obtain the resistivity constant as indicated in Sec. 2, and repeat this procedure 50 times for each configuration, for about 100 independent configurations as ensembles.

Let us first take a look at the results for pure gravity (Fig.11). We find that peaks get narrower as the size grows. The value $r \approx 2.6$ should be compared to $\sqrt{3}$ of the flat network(i.e. a honeycomb lattice). An increase in $r$ is understood to be a reflection of the fractal nature of the surface. As the surfaces get larger, finite size effects due to the discreteness diminish and eventually the peak will grow indefinitely. This is what we have expected as the continuum limit of a network of resistors. We find similar tendency in the one scalar case $(c=1)($ Fig,2). Also, in Fig, 3, we

\footnotetext{
1 There are analogies between Feynman diagram and network of resistors: ( $\alpha_{l} \leftrightarrow$ resistance), $\left(x_{i} \leftrightarrow\right.$ voltage at i), ( $p_{l} \leftrightarrow$ current through 1$)$ and ( $Q \leftrightarrow$ heat generation).
}

show data to justify the assumptions of the analog model for the case of pure gravity. Each resistance is distributed randomly with weight $e^{-\alpha_{l}}$. These distributions indicate the same behavior with previous cases $c=0$. In Fig. 1, f we plot the distributions of $r$ for the $c=2,3$ cases. Here no sharpening of peaks with increasing sizes is seen unlike the previous cases, which suggests that the surface does not approach a smooth continuum limit. This is considered as the reflection of the tachyonic mode instability in the continuous theory. The same tendency is also supported in the case of 3 critical Ising spins.

In conclusion we have observed the so called $c=1$ barrier for two dimensional quantum gravity coupled to matter as the transition from a well-defined complex structure to an ill-defined one.

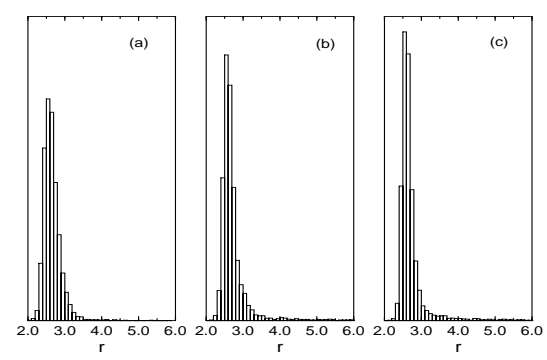

Figure 1. Resistivities for the case of pure gravity. The size of $(a),(b)$ and $(c)$ are 4000, 8000 and 16000 triangles respectively.

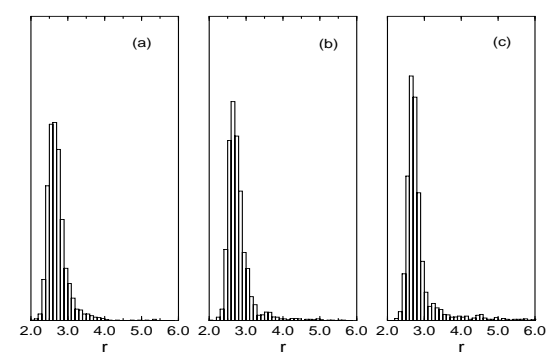

Figure 2. Resistivities for the case of 1 scalar.

\subsection{Separating the conformal modes}

Next, we discuss how to extract information on the conformal mode. Once we have found the 


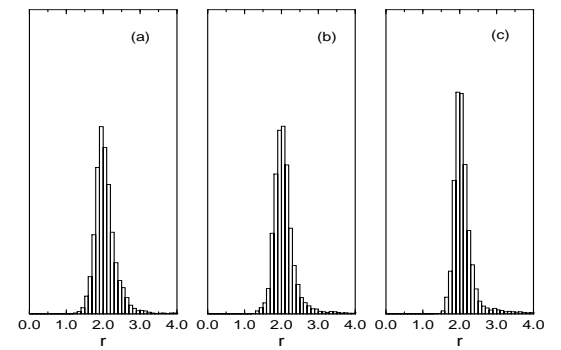

Figure 3. Resistivities for the case of pure gravity when each resistance is randomly distributed.

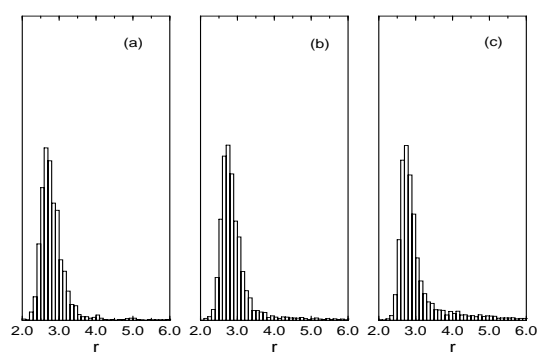

Figure 4. Resistivities for the case of 2 scalars.

value of resistivity, we can assign a complex coordinate to each vertex of the dual graph from eq.(4) and eq.(5). In Fig.6 we plot the distribution of coordinates for all the vertices of a surface for the case of pure gravity with 8000 triangles. The point density around a point $z$ should be proportional to $\sqrt{g(z)}$, because each vertex is supposed to carry the same space-time volume. In the conformal gauge which we have been employing, we have $\sqrt{g(z)}=\exp \phi(z)$, where $\phi(z)$ is the conformal mode. This argument may be over-simplified in the sense that we did not pay attention to the quantum fluctuation of $\phi(z)$ and the renormalization of the composite operator $\exp \phi(z)$. What we can say safely is that the point density at $z$ after taking an ensemble average should be equal to the four-point function

$$
\lim _{w \rightarrow \infty}|w|^{4}<e^{\alpha \phi(0)} e^{\alpha \phi(1)} e^{\alpha \phi(w)} e^{\alpha \phi(z)}>,
$$

in the Liouville theory, where $w$ is a point on the complex plane and $\alpha$ is the renormalization factor for the conformal mode. In a recent paper 河,

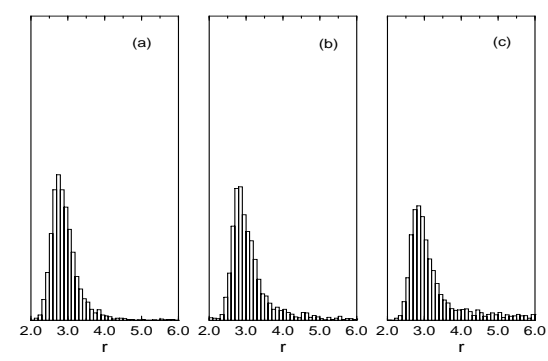

Figure 5. Resistivities for the case of 3 scalars.

an estimate for this four-point function has been given. This should be compared with Fig.6.

\section{Acknowledgements}

We are grateful to N.D.Hari Dass and N.Ishibashi for useful discussions and comments. One of the authors(N.T.) is supported by Research Fellowships of the Japan Society for the Promotion of Science for Young Scientists.

\section{REFERENCES}

1. N.Tsuda and T.Yukawa, Phys.Lett. B305 (1993) 223;

H.Kawai, N.Kawamoto, T.Mogami, and Y.Watabiki, Phys.Lett. B306 (1993) 19.

2. B.Sakita and M.A.Virasoro, Phys.Rev.Lett. 24 (1970) 1146;

H.B.Nielsen and P.Olesen, Phys.Lett. B32 (1970) 203.

3. See for example J.D.Bjorken and S.D.Drell, McGraw Hill, New York, 1965.

4. A.B.Zamolodchikov and Al.B.Zamolodchikov hep-th/9506136 